\title{
Human Immunodeficiency Virus Cases on the Rise in Pakistan
}

\author{
Tahir Sultan Shamsi* \\ Department of Clinical Hematology, National Institute of Blood Disease \& Bone Marrow Transplantation, Karachi, Pakistan.
}

Human immunodeficiency virus infection leads to acquired immune deficiency syndrome. After horizontal transmission (through unprotected sex, contaminated blood transfusion or sharing contaminated syringes among main line drug addicts) or vertical transmission (from mother to foetus during pregnancy or during deliver / breast feeding), the person remains asymptomatic for years or may develop flu like symptoms. HIV remains quiescent in the monocytes and helper $\mathrm{T}$ cells interfering with and knocking out host's immune system immensely increasing the risk of opportunist and non-opportunist infections as well as various malignancies. There is no cure for HIV or a vaccine available as of today; current antiretroviral treatment slows the course of the disease and may lead to a near-normal life in affected individuals. Treatment is recommended without delay; without treatment, the affected individuals don't survive beyond first decade.

In 2016, there were 36.7 million HIV affecties that culminated in 1 million deaths. Approximately 300,000 fewer new HIV cases reported in 2016 than in 2015. Most cases reported from sub-Saharan Africa. From early 1980s till now, an estimated 35 million deaths were reported worldwide.

On 25 April 2019, Pakistani media reported hundreds of HIV infected children in Talukas Rattudero of Larkana district of Sindh province. An immediate action was to set up population screen for general population in the area. HIV rapid test kits were used for this purpose. In subsequent three months, a total of 30,192 people were screened, of which 876 were found positive. Eighty-two per cent $(719 / 876)$ of these were below the age of 15 years. During this exercise, following risk factors were identified: unsafe intravenous injections by medical caregivers; unsafe practices during delivery of foetuses; poor screening at blood banks; non-existent infection control programmes; and improper collection, storage, segregation and disposal of hospital waste.

This was the fourth reported outbreak of HIV in this district in last two decades. In 2003, the first outbreak was among IV drug users, the second one was among 12 paediatric patients at a paediatric hospital in 2016, and the third one, a massive clustering of HIV among 206 patients in a dialysis unit was reported.

Pakistan is one of the countries in the WHO Eastern Mediter-

*Address correspondence to this author at the Department of Clinical Hematology, National Institute of Blood Disease \& Bone Marrow

Transplantation, Karachi, Pakistan. E-mail: t.shamsi.62@gmail.com ranean Region where new HIV infections are increasing at an alarming level since 1987. The current HIV epidemic in Pakistan is defined as a concentrated epidemic. Although the overall prevalence is still less than $1 \%$ in the adult population, the latest estimate (2017) of people living with HIV was 150,000. Last year, 21,000 new HIV cases were recorded.

When we review the healthcare facilities in smaller towns and in rural areas; the situation is more or less similar. There is sharp contrast of available healthcare for different segments of our society. One extreme, available blood products from voluntary blood donors are screened using nucleic acid testing, the other extreme is no or very poor screening from professional blood donors. In the middle, sub-optimal blood product screening is done. Although government regulatory bodies regulates mushroom blood banks but standardisation of blood screening is non-existent. Regional blood centres (RBCs) only recently established in the country, but their impact on the practices of blood transfusion practices remains yet to be seen. Till then, blood transfusion transmitted infections will remain a major risk and a route for spread of HIV in the country.

Use of narcotics and other substances is rampant in our society. Sharing hypodermic syringes among IV drug abusers is found to be high in the country. This practice is considered an established mode of spread of HIV and other viruses. Sharing syringes by the medical facilities was found to be an important determinant in the current HIV epidemic in Larkana. Both situations are unsafe but syringe-sharing practice by healthcare professionals is a criminal negligence and should be stopped.

Unsafe sexual practices are by no means less common in different segments of the society. Prisoners, Eunuchs, sex workers, and using children as sex objects are some of the disturbing practices. Sex-education, public awareness about the risks involved and poverty alleviation are effective ways of reducing the spread of HIV.

Coming back to the current epidemic reported this year, WHO and local teams found the following facts:

- Scarcity of correct information to determine the magnitude of the event.

- Mostly children under 5 years age group were affected.

- Date / period of exposure of HIV to the cases is unknown.

www.njhsciences.com 
- Lack of information regarding all possible sources of exposure.

- Lack of appropriate ARV drugs.

- Why repeated HIV outbreaks have occurred in the same geographical area.

This Larkana event might be the tip of the iceberg. Thousands of thalassaemic children receive blood transfusion every day in the country. Sporadic HIV cases are identified regularly from districts of all provinces. Repeated blood transfusion exposes these kids to the risk of acquiring not only HIV but the data suggest seroprevalence of HCV and HBV to be as high as $60 \%$ and $20 \%$ respectively. Further epidemiological work is needed which will help identify the magnitude of the problem, and whether this event was isolated in nature, or a long existing problem with these accidentally diagnosed cases representing the tip of the iceberg of a larger epidemic.

After this event in Larkana, WHO issued the following advice. We are quoting it as such:

This event highlights the importance of using high-impact interventions to reduce vulnerability and prevent transmission mainly in health care settings. It also takes into consideration the prevention of sexual transmission in high risk groups, transmission through injecting drug use and mother-to-child transmission.

WHO recommends that after 18 months of age, three different assays may be required to establish the diagnosis of HIV infection. However, infants less than 18 months of age who are born to HIV infected mother should be diagnosed through nucleic acid testing (NAT).

WHO stresses the importance of immediately linking all those diagnosed with HIV infection to antiretroviral treatment (ART), where the test should be repeated to rule out errors in diagnosis (in case second test is negative) and, thereafter, ART should be started without any delay.

\section{CONFLICT OF INTEREST}

Declared none.

\section{ACKNOWLEDGEMENTS}

Declared none.

\section{SUGGESTED READING ON THIS ISSUE}

[1] Adnan Ahmad Khan, Ayesha Khan. The HIV epidemic in Pakistan. J Pak Med Assoc 2010; 60: 300-7.

[2] https://www.who.int/csr/don/03-july-2019-hiv-cases-pakistan/en/

[3] https://en.wikipedia.org/wiki/HIV/AIDS_in_Pakistan

[4] WHO. Report on the global HIV/AIDS epidemic-June 1998: Available at: data.unaids.org/pub/report/1998/19981125_global_epidemic_report_en.pdf

[5] UNAIDS. Pakistan country profile. 2018; Available at: unaids.org/en/regionscountries/countries/pakistan

[6] WHO. Consolidated guidelines on HIV testing services. 2015; Available at: who.int/hiv/pub/guidelines/hiv-testing-services/en/

[7] WHO. Consolidated guidelines on the use of antiretroviral drugs for treating and preventing HIV infection. second ed. 2016; Available at who.int/hiv/pub/arv/arv-2016/en/

[8] Ansari SH, Shamsi TS, Khan MT, et al. Seropositivity of hepatitis C, hepatitis B and HIV in chronically transfused $\beta$-thalassaemia major patients. J Coll Physicians Surg Pak 2012; 22: 610-1

[9] Shah T, Hussain W, Ali N, et al. Frequency distribution and risk factors of hepatitis $B$ virus and hepatitis $C$ virus infections among thalassemia patients: a regional study. Eur J Gastroenterol Hepatol 2019; 31: 248-252. 